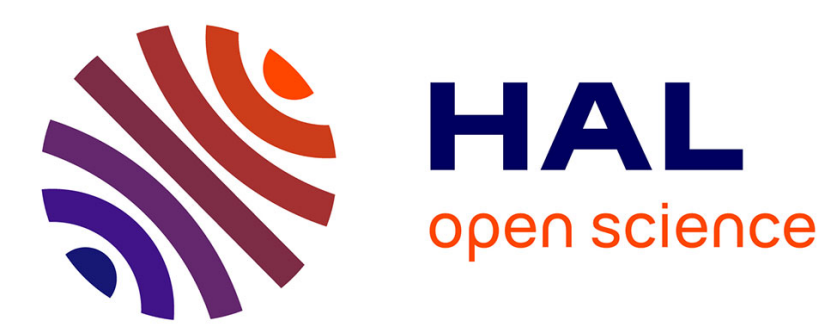

\title{
Lithium-niobate-based Cavity Resonator-Integrated Guided-mode Resonance Filters
}

\author{
Stéphane Calvez, Antoine Monmayrant, Olivier Gauthier-Lafaye
}

\section{To cite this version:}

Stéphane Calvez, Antoine Monmayrant, Olivier Gauthier-Lafaye. Lithium-niobate-based Cavity Resonator-Integrated Guided-mode Resonance Filters. Conference on Lasers and ElectroOptics/Europe, Jun 2019, Munich, Germany. 2019. hal-02296330

\section{HAL Id: hal-02296330 \\ https://hal.laas.fr/hal-02296330}

Submitted on 25 Sep 2019

HAL is a multi-disciplinary open access archive for the deposit and dissemination of scientific research documents, whether they are published or not. The documents may come from teaching and research institutions in France or abroad, or from public or private research centers.
L'archive ouverte pluridisciplinaire HAL, est destinée au dépôt et à la diffusion de documents scientifiques de niveau recherche, publiés ou non, émanant des établissements d'enseignement et de recherche français ou étrangers, des laboratoires publics ou privés. 


\section{Lithium-niobate-based Cavity Resonator-Integrated Guided-mode resonance Filters (CRIGFs)}

\section{S. Calvez, A. Monmayrant, O. Gauthier-Lafaye}

\section{Introduction}

\section{Context}

- Guide-Mode Resonance Filters (GMRFs - review paper ${ }^{1}$ ):

- Spectral filters with less complex layer structure than thin-films

- Q up to $10^{4}$

- Angularly tunable response

- Demonstrated enhancement of nonlinear interactions but with large beams ${ }^{2-4}$

- Cavity Resonator Integrated Guided-mode Resonance Filters (CRIGFs)

- $\mathrm{CRIGF}=$ GMRF section in a cavity ${ }^{5}$

Spatial (mode) localisation $(\sim 1-100 \mu \mathrm{m})$ Q up to $10^{3}$

Wider angular acceptance than GMRF $^{6}$

$x$ Spectrally-fixed filters

Used as wavelength-stabilizing filters in extended-cavity diode lasers ${ }^{7-9}$

\section{This work}

- Fabrication of CRIGFs on a material platform compatible with nonlinear interactions - Demonstration of tunability
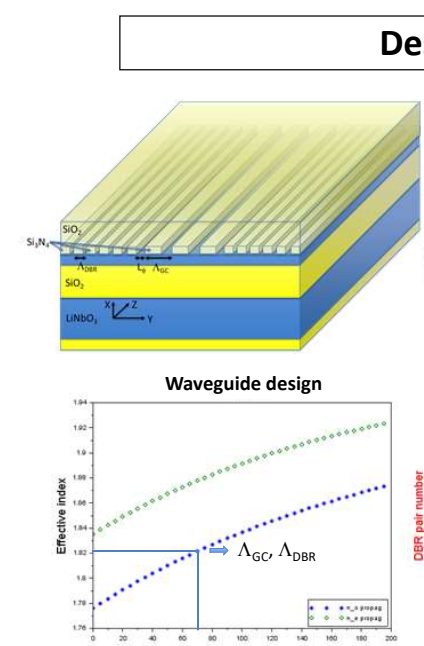

GC design as GMRF

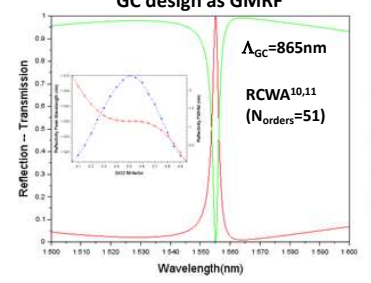

\section{Design}

AR coating design

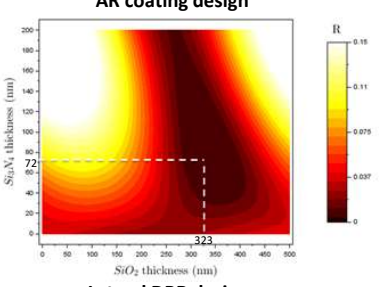

Lateral DBR design

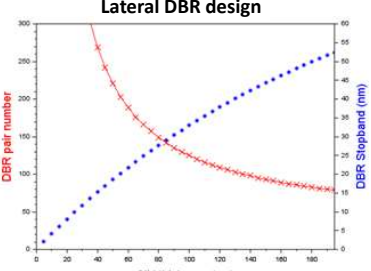

SiN thickness (nm)
CRIGF

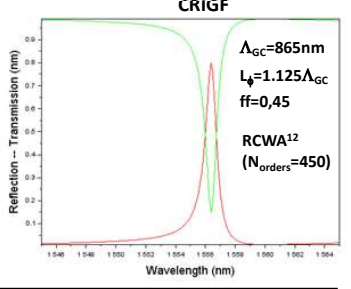

Creating master mask in

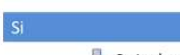

Si Resist deposition

$\sqrt{\text { e-beam Lithography }}$

If oreching

Dry etching

$\mathrm{Si}$

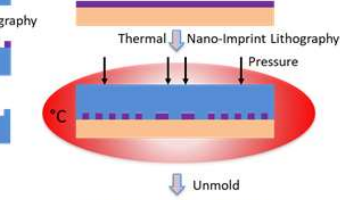

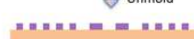

\section{References}

G. Quarantra et al, Laser Photon. Rev., 12 (9), 180017 (2018)

A. Saari et al, Opt. Express, 18 (12), 12298 (2010)

J.H. Lin, Opt. Express, 22 (3), $2790(2014)$

J. Inoue et al., Appl. Phys. Express, 5, 022201 (2012)

X. Buet et al., Opt. Express, 20 (12), 9322 (2012)

X. Buet et al., Electron; Lett., 48 (25), 1619 (2012)

A. Monmayrant et al, ECLEO 2019, paper CB-7.4 + Booth B2.401

A. Monmayrant et al, ECLEO2019, poster CB-P1 + Booth B3.421/4

1. M.G. Moharam et al, J. Opt. Soc. Am. A, 12 (5), 1068 (1995)

12. P.C. Chaumet et al, Opt. Lett., 41 (10), $2358(2016)$

3. L. Moretti, J. Appl. Phys., 98(3), 036101 (2005)

4. A. Arbabi et al, Opt. Lett., 38(19), 3878 (2013)
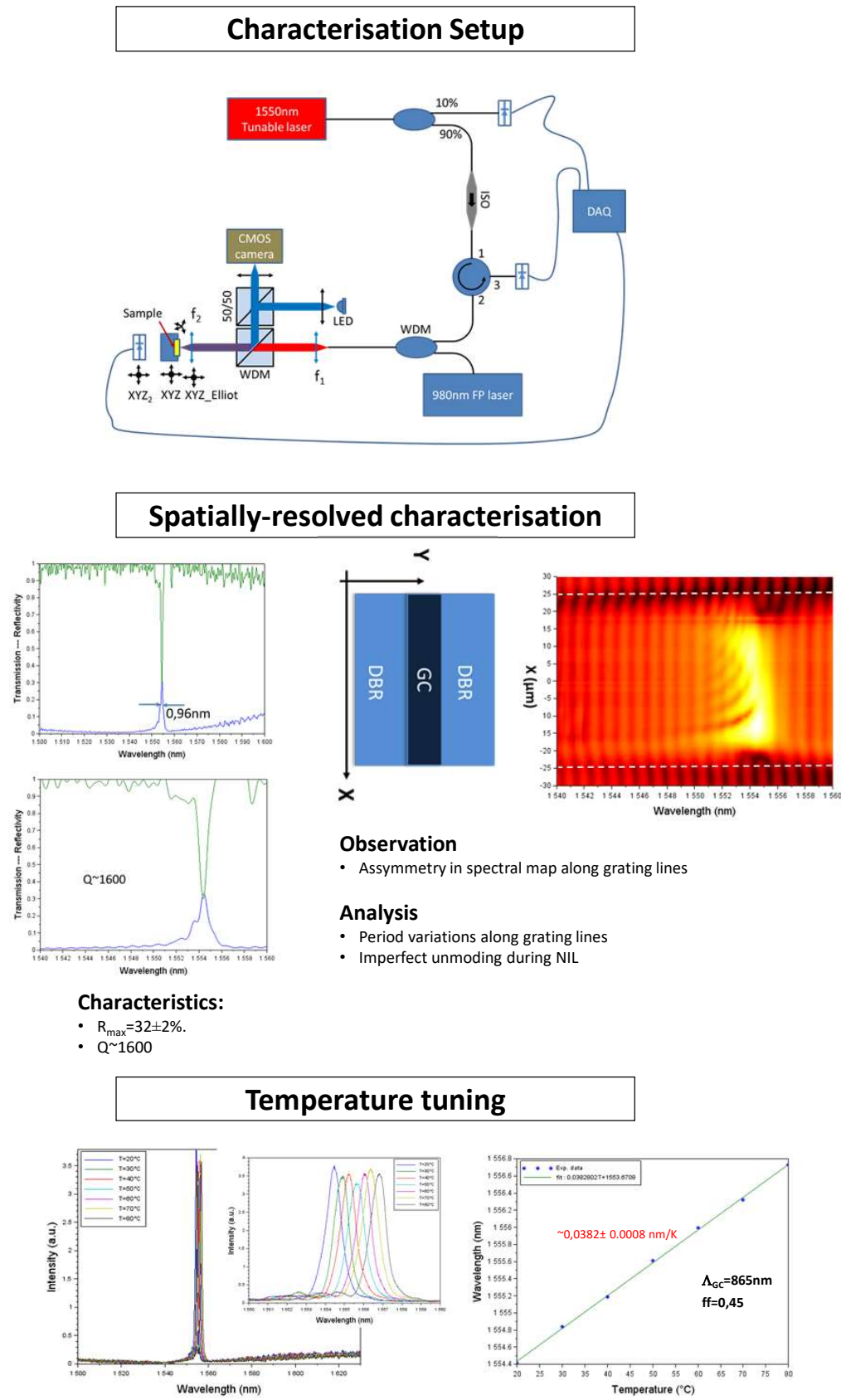

Analysis: Parameters ${ }^{13,14}: \frac{\partial n_{L N}(1550 \mathrm{~nm})}{\partial T}=3.310^{-5} / \mathrm{K}$ $\frac{\partial n_{S i N}(1550 \mathrm{~nm})}{\partial T}=2.4510^{-5} / \mathrm{K}$ $\begin{gathered}\frac{\partial T}{\partial n_{\text {SiO } 2}(1550 \mathrm{~nm})} \\ \partial T\end{gathered}=0.9510^{-5} / \mathrm{K}$ $\alpha_{T, \text { subs }}=0.7510^{-5} / \mathrm{K}$

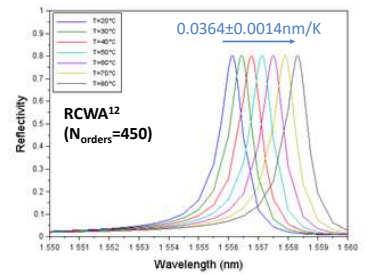

\section{Conclusions}

First demonstration of CRIGF on Lithium Niobate On Insulator (LNOI)

Characteristics : $R_{\max }=32 \pm 2 \%$ and $Q \sim 1600$ à $1554.5 \mathrm{~nm}$

for 8.65- $\mu \mathrm{m}$-wide grating-coupler structure

Performance affected by NIL unmolding step

Thermally tunable response over $1.2 \mathrm{~nm}$ with resonance shift rate of $0.038 \mathrm{~nm} / \mathrm{K}$

Tuning characteristics reproduced numerically using reported parameters

Future work

- Devices with integrated heaters / electro-optic tuning 